International Journal of Man-Machine Studies (1969) 1, 333-360

\title{
Linear and Nonlinear Models of the Human Controller
}

\author{
Brian R. Gaines \\ Department of Electrical Engineering Science \\ University of Essex, Colchester, Essex, U.K
}

This paper presents a review of recent studies of the human controller both in psychology and in control engineering. Theoretical and technological problems in the study of skilled behaviour are first discussed, and the desirable constraints upon any "model" are outlined. The foundations of linear continuous modelling of the human controller and experimental data on the validity and utility of linear models are then reviewed. The evidence for nonlinear and discontinuous behaviour in the human controller is then outlined, and studies of non-linear models based on modern optimal and sampled-data control theory are then presented.

\section{The Study of Perceptual-motor Skills}

Although human cognitive skills and verbal behaviour are generally assumed to involve higher level processes and to be more complex than human perceptual-motor behaviour (for example, Bartlett (1958) has suggested that the analysis of human problem-solving behaviour is simplified by regarding it as a "high-level skill"), in practice a far greater effort has been applied to the study of the cognitive skills involved in learning and remembering verbal material, and in problem-solving, than to the perceptual-motor skills involved in such tasks as flying, driving and typing. Part of this emphasis stems from the philosophical origins of psychology and the associated emphasis on studies of human conscious thought processes; but the continued relative neglect of perceptual-motor skills must have a more fundamental cause, since it would seem reasonable for a logical development of experimental psychology to treat the problems of perceptual-motor co-ordination involved in driving and flying, before investigating the far more complex problems of verbal behaviour and problem-solving.

There are two main reasons for this disparity in the amount of effort expended on studies of human cognitive skills on the one hand, and perceptual-motor skills on the other. First, perceptual-motor skills cannot generally be verbalized and introspective analysis is misleading, if not impossible. Although, in modern experimental psychology, introspection is discounted as a source of information about human behaviour, in practice it is an important source of hypotheses and experimental designs, even if reference to it is carefully eradicated from published results. Although there is considerable coupling between verbal instructions and the learning, and performance, of perceptual-motor skills, this is not so strong as in cognitive skills more closely related to the use of language, and the lack of verbalization deprives the worker on perceptualmotor skills of two major sources of information-his own experience, and the introspective analysis of others.

The second reason for a disparity of effort and achievement between studies of cognitive skills and perceptual-motor skills is, to a large extent, technological. Perceptual-motor skills are externally paced and the operator is essentially on-line, with the detailed time-patterns of his visual inputs and manual outputs being a major aspect of the skilled performance. To record these time patterns accurately, recorders with a bandwidth of several hundred Hertz are required, and these only became readily available in the early fifties. To analyse the data in these 
recordings, powerful computational facilities are required, and these became readily available only in the late fifties (analog computers) and early sixties (digital computers). To control the experimental situation in which a perceptual-motor skill is being observed, it is necessary to perform the data analysis rapidly during the experiment-that is, with an on-line, real-time system. Unfortunately, whilst analog computers have always been available for real-time use, they are essentially linear, continuous-time systems which have proved inadequate for the analysis of the nonlinear, discrete-time behaviour of the human controller, and on-line digital computers for the control of psychological experiments are only now, in the late sixties, becoming available. One effect of this requirement for complex technology has been that most notable studies of the human controller have taken place in a military context, and the highly skilled aircraft pilot has, for example, been far more studied than the normal or brain-damaged individual.

Thus, the concentration of effort in experimental psychology on the macroscopic aspects of cognitive skills, rather than the microscopic aspects of perceptual-motor skills, has been determined both by the availability of a natural language for describing, and communicating about, cognitive skills, and by the lack of suitable instruments for measuring and analysing behaviour in perceptual-motor skills. Recent advances in technology have made available the online digital computer (Miller, Bregman \& Norman, 1965) as a suitable instrument for the control of experiments on perceptual-motor skills, and the modelling of skilled behaviour; recent advances in the study of nonlinear, discrete-time control systems are making it possible to overcome some of the methodological problems in the study of skilled behaviour; and hence our knowledge of human perceptual-motor behaviour may be expected to progress rapidly during the next decade.

This paper reviews studies of the human controller, mainly in the single-input, single-output, "compensatory" tracking situation, and discusses the validity and utility of models of human behaviour based on these studies. In the following section some methodological problems in modelling human behaviour are discussed. Then linear continuous models of the human controller based on "describing-function" measurements are critically reviewed, followed by nonlinear models based on modern optimal and sampled-data control theory.

\section{Methodological Problems in Modelling the Human Controller}

The human being is a very complex organism whose range of behaviour encompasses not only skilled control tasks, but also instinctive and emotional reactions, such as those resulting from pain or fear, and linguistic communication with other humans. Language, both natural and scientific, is inadequate to describe the majority of human behaviour in all but a cursory way. Natural language has developed to serve the objectives of human society, particularly those objectives which require communication between individuals. The descriptive power of the language, and those properties of behaviour which are abstracted for description, reflect this function of language; for example, behaviour tends to be described in terms of its end result rather than as a movement pattern. Scientific language, particularly the purely mathematical description of the human operator: first, as a mechanical system - a "multi-degree of freedom" object made up of jointed solids; and second, an electrochemical system-the blood supply, nervous system, and so on-is possible to apply in theory, but impossible in practice. Not only are there practical difficulties in the measurement of the number of variables involved, and problems of data-analysis in that each behaviour becomes a unique event, but the analysis, 
representation and comprehension of one system requires a meta-system which is considerably larger than the system represented, and the human brain cannot act as an adequate meta-system for a complete representation of itself.

Thus, whatever approach is taken to the description and modelling of human behaviour, it is bound to be only a partial approach, or approximation, and in practice only comparatively small domains of behaviour can be modelled in any depth. It has been customary to delimit these domains by splitting human activities into various categories-conditioning, problem-solving, verbal behaviour, perceptual-motor skills, and so on (Melton, 1964a), but even within these categories no reasonably comprehensive models of behaviour are available, and there are crosscategory similarities which are stronger than within-category differences (Melton, 1964b). In these circumstances, it seems reasonable not to start with pre-conceived categories of behaviour, but rather to evaluate a model by the size, and importance, of the domain of behaviour for which it is an adequate representation.

Models capable of representing behaviour over large domains are particularly important in the study of learning systems where the mode of behaviour is expected to vary widely with experience. A variety of models is required, and within the modelling schemata there must be scope for a sufficient variety to provide adequate matches during all phases of learning. Moreover these models should be related to one another and there should be a smooth progression through related models during learning, rather than a succession of sudden transitions from one model type to another. Equally, changes in the environment should not involve sudden, major changes in the model of the controller-if the model is "correct", any changes in it due to changes in the environment will be the result of learning, and can be expected to be fairly gradual.

These requirements may be formalized mathematically, and, indeed, future advances in the study of human behaviour may be crucially dependent on an adequate formalization. However, none yet exists, and the present paper reviews studies of human skill within their intrinsic frameworks, whilst attempting to indicate the nature of the broader framework in which they may be placed.

\section{Linear Models of the Human Controller}

Linear modelling of the human operator performing a control task is of interest for several distinct reasons: first, it is an example of the "black-box", cybernetic approach to modelling a system by a structure based on its behaviour; second, linear systems are one of the most important families of control policies, in that their properties are virtually completely known, and hence no mathematical problems arise within the strict framework of the model itself, and full advantage may be taken of the control engineering studies of linear controllers and their behaviour; third, there have been many detailed experimental studies of linear models of the human controller in a variety of situations, and any further developments must take into account the data gathered in these studies.

In the following sub-sections, the theoretical basis and implications of linear modelling are first established, the main results of experimental studies are outlined, the utility of the linear model and the "meaning" of its parameters and "goodness-of-fit" are discussed, and the application of linear models, particularly in studies of learning, is evaluated. 


\subsection{The foundations of linear modelling}

In mathematical terms, a linear transformation is a mapping between vector spaces which obeys the superposition principle, in that the transform of the sum of two vectors is the sum of the transforms of each of the two individual vectors (Mirsky, 1955). Functions of time over an interval form a vector space which is infinite-dimensional, and the operations of addition, scaling, integration, differentiation and time-delay may be shown to be linear operations (Riesz \& Nagy, 1955). A linear dynamical system is one whose action may be represented in terms of these operations alone (Birkhoff, 1927), and these systems have been extensively studied in linear systems theory. In particular, linear functionals from the space of linear functions to a complex algebraic variable have been developed, such as the Laplace transform which enable linear operators on time-functions to be manipulated in an algebraic manner with full mathematical rigour.

Because linear system theory is so well-developed and contains such a powerful body of techniques for studying system behaviour, when a nonlinear system is to be analysed it is convenient to attempt to approximate its behaviour by that of some linear system. If the linear approximation is, in some sense, good then much of the behaviour of the nonlinear system may be predicted from a linear model whose behaviour is readily determined. In control engineering, techniques have been developed for the analysis of the stability of nonlinear systems using a linear approximation, or "describing function" (Gibson, 1963). The first techniques developed were based on an analysis of the behaviour of the nonlinear system when excited by simple harmonic waveforms at various frequencies. Booton (1953) extended these results to systems excited by noise-like signals, and it is his technique which has been used to derive linear approximations to human control policies.

A detailed mathematical analysis of the describing function technique is not relevant to the present studies, but certain assumptions made, and their applicability to the human controller, are important in evaluating the utility and implications of linear models of the human operators, and these assumptions will be outlined here. The configuration envisaged for linear modelling is shown in Figure 1: a nonlinear system, N, drives a linear system, G, the output of which, c(t), is subtracted from the input signal, $\mathrm{r}(\mathrm{t})$, and fed to the non-linear system as an error signal, $\mathrm{e}(\mathrm{t})$; the output of the nonlinear element, $\mathrm{m}(\mathrm{t})$, is assumed to be made up of two components, one of which is correlated with the error, and the other of which $n(t)$, is independent of it.

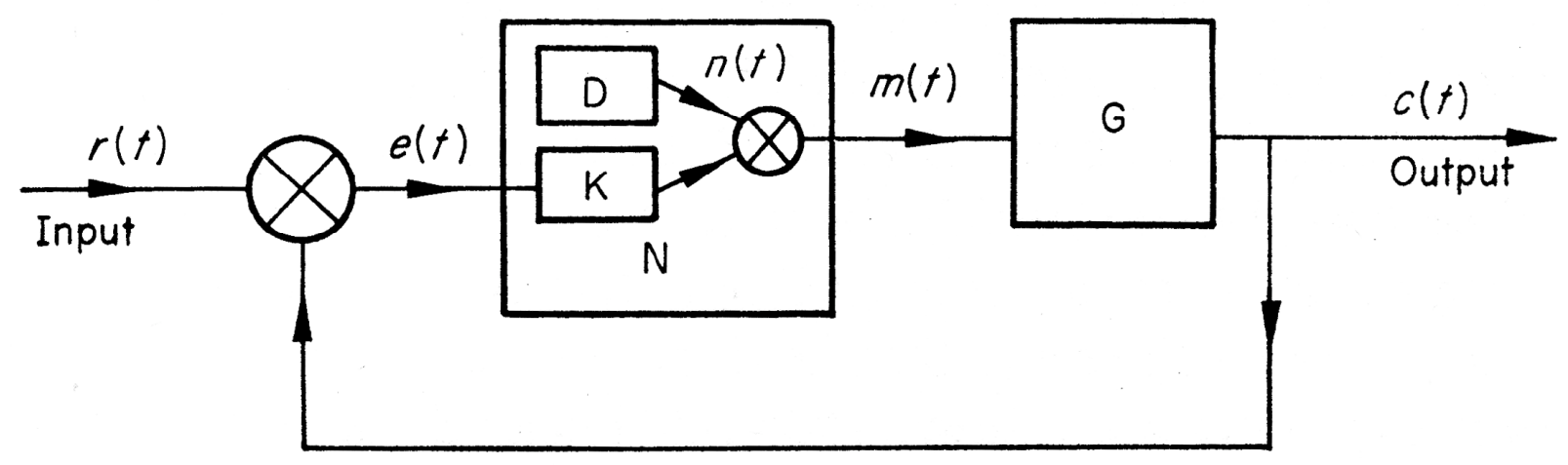

Figure 1. Linear analysis of nonlinear feedback system

Booton's analysis depends on the assumption that the signals in the system, particularly e (t), are Gaussian processes. Even if $\mathrm{e}(\mathrm{t})$ is Gaussian, $\mathrm{m}(\mathrm{t})$, the output of the nonlinear element, will not 
be, and hence neither will $c(t)$. However, if the linear element $G$ is narrow-band with respect to the input spectrum, by the central limit theorem its output will more closely approximate a Gaussian process (Gibson, 1963, p. 387), and hence, if $r(t)$ is Gaussian, then so will be $e(t)$. Thus, the use of describing function techniques to model a nonlinear system is dependent on the assumptions that the input is Gaussian, and that the nonlinear element is followed by a filter which is narrow-band with respect to the input spectrum.

There are alternative views of the describing function technique which throw some light on the meaning of the assumptions made. The overall linear model is an approximation to the transfer function between the input, $r(t)$, and the output $c(t)$. This will be good to the extent that $G$ is a narrowband filter which eliminates frequencies, $n(t)$, which are not present in the input. Since $G$ is known, $\mathrm{K}$, the linear model of the nonlinear element, is effectively available from the closedloop response. However, the relationship between $\mathrm{e}(\mathrm{t})$ and $\mathrm{m}(\mathrm{t})$, predicted from a knowledge of $\mathrm{K}$, will only account for that part of $\mathrm{m}(\mathrm{t})$ which is not filtered out by G. Hence, to the extent that $\mathrm{G}$ is narrow-band and enables the describing function technique to be used, it also restricts the model of $\mathrm{N}$ to account for only a small part of the behaviour of the nonlinear element.

A further effect on the type of linear model obtained for $\mathrm{N}$ is dependent on the amplitude of $\mathrm{e}(\mathrm{t})$ compared with that of $r(t)$ (more strictly on the ratio of r.m.s. amplitudes). Since $n(t)$ and $r(t)$ are uncorrelated random processes, any part of $\mathrm{n}(\mathrm{t})$ which passes through $\mathrm{G}$ and is fed back to form $\mathrm{e}(\mathrm{t})$ increases the error, on average. Hence, for the controller to perform well and maintain a small error between overall input and output, it is necessary for the nonlinearly generated part of its output which passes through $G$ to be small. In the context of the human controller, this implies that a good linear model may be obtained for the overall loop behaviour of an operator controlling a linear system; the model will not account for any components of the operator's output which have little effect on the system.

The linear approximation to a nonlinear system varies with system variables, such as the mean amplitude of the input - for example, a relay switching function whose output is the sign of its input has a constant r.m.s. output, and hence its "equivalent gain" is inversely proportional to the r.m.s. input amplitude. Similar dependencies on e(t), and hence on both $\mathrm{r}(\mathrm{t})$ and $\mathrm{G}$, occur for any form of nonlinear element, $\mathrm{N}$, and the measured describing function will be found to be a function of the input and controlled element. Thus $\mathrm{N}$ will appear to be "adaptive" to the input and controlled element, but this "adaption" is unrelated to any effort by $\mathrm{N}$ to improve its performance-the "adaption" is an artefact resulting from the linear modelling, rather than adaptive behaviour on the part of $\mathrm{N}$.

To conclude this critical examination of the describing function technique, it is worth quoting Gibson's (1963, p. 388) remark that, "under a wide set of circumstances the use of the Gaussian describing function to compute closed-loop response is invalid." Although the technique is based on a mathematical analysis which looks both impressive and plausible, in practice its derivation is based on highly restrictive assumptions, and, even when these apply, the meaning of the results obtained is not clear. Although this critique has been largely destructive, it is essential to consider linear modelling in some depth because it is the most obvious, and most readily applied, technique for analysing human control policies. Equally, any other approach to modelling must be able to withstand similar criticism, and the defects of linear modelling can be most readily overcome if they are thoroughly analysed. 


\subsection{Results of linear model studies of the human controller}

The earliest study of the human operator as a linear servomechanism is that of Tustin (1947) who proposed that, despite amplitude nonlinearities, temporal discontinuities and haphazard fluctuations, there might be an "appropriate linear law" that would describe the main part of the operator's behaviour. Since that time, there have been many studies, including those of Russell (1951), Krendel (1951, 1952), Elkind (1956) and McRuer and Krendel (1959). The early studies have been reviewed by Licklider (1960), and more recent reviews have been given by Summers \& Ziedman (1964), Young and Stark (1965) and McRuer, Graham, Krendel and Reisener (1965). Hall (1963) has published a concise study covering the main aspects of linear models of the human operator in a flying situation, and this is summarized here.

All the studies of linear models reference have taken control situations in which it is reasonable to expect the human operator to act linearly: the error, $\mathrm{e}(\mathrm{t})$, has been displayed on an analogue, positional display, such as an oscilloscope or pointer-meter; the operator's output $m(t)$ has been applied to an analogue positional control, such as a joystick. In most cases only the error, e(t), has been displayed to the operator (compensatory tracking), but in a few studies, Elkind's in particular, $r(t)$ and $c(t)$ have been displayed on the same scale (pursuit tracking). Hall studied compensatory tracking with $\mathrm{G}$, the controlled element, having a form corresponding to the short period motion in the longitudinal dynamics of aircraft-its transfer-function was of the form:

$$
G(s)=\frac{L(1+0.6 s)}{s\left(1+\frac{2 k}{w_{n}} s+\frac{1}{w_{n}^{2}} s^{2}\right)}
$$

where $\mathrm{L}$ is the gain of the controlled element, $\mathrm{w}_{\mathrm{n}}$ is its undamped natural period in rad/sec, and $\mathrm{k}$ is the damping ratio; in Hall's experiments, $0 \leq \mathrm{k} \leq 1$ and $0 \leq \mathrm{w}_{\mathrm{n}} \leq 7$. The input, $\mathrm{r}(\mathrm{t})$, was a Gaussian random signal passed through a low-pass filter of the form $1 /(1+\mathrm{s})^{3}$. Hall's operators were highly skilled pilots, used to controlling elements with dynamics of this form.

Hall's main results are illustrated in Figure 2 by plots of various variables in a plane with damping ratio, $\mathrm{k}$, as abscissa, and undamped natural frequency, $\mathrm{F}_{\mathrm{n}}=\mathrm{w}_{\mathrm{n}} / 2 \pi$, as ordinate. He considered the operator to be acting linearly if the r.m.s. level of the "remnant" term, $n(t)$, was less than 5\% of the r.m.s. operator output, $m(t)$. From Figure 2(a), it may be seen that the operator acted linearly, by this criterion, for the higher values of natural frequency and damping ratio. Figure 2(b) shows contours delimiting regions of similar tracking performance in terms of mean error.

Hall found radical changes in the form of linear model associated with different controlled elements, and the regions associated with different models are delimited in Figure 2(c); the forms of model are given in Table 1. The term $\mathrm{e}^{02 \mathrm{~s}}$, occurring in all four models, is a pure time delay of $200 \mathrm{msec}$ similar in magnitude to a simple visual/motor reaction-time. The terms in $\mathrm{s}$ in the numerators of the transfer-functions correspond to a phase-lead, or dependence upon the input velocity, and similar terms in the denominator correspond to a phase-lag, or smoothing of the error signal. Region A is one of high damping and medium-speed response, and the model has a predominant lead term showing that the operator is using the velocity of the error to predict ahead. Region $\mathrm{C}$ is one of low damping and fast response, where the higher frequency components of the input are very apparent, and the model has a dominant lag term showing that the operator is filtering out, or responding less, to these components. In Region C, between these 
two, the model suggests that the operator, apart from his reaction-time delay, is acting as a pure gain element. In region D, where the system is slow and underdamped, second-order terms in $\mathrm{s}$ appear in the numerator, showing that the operator is now making use of information about the acceleration of the error.

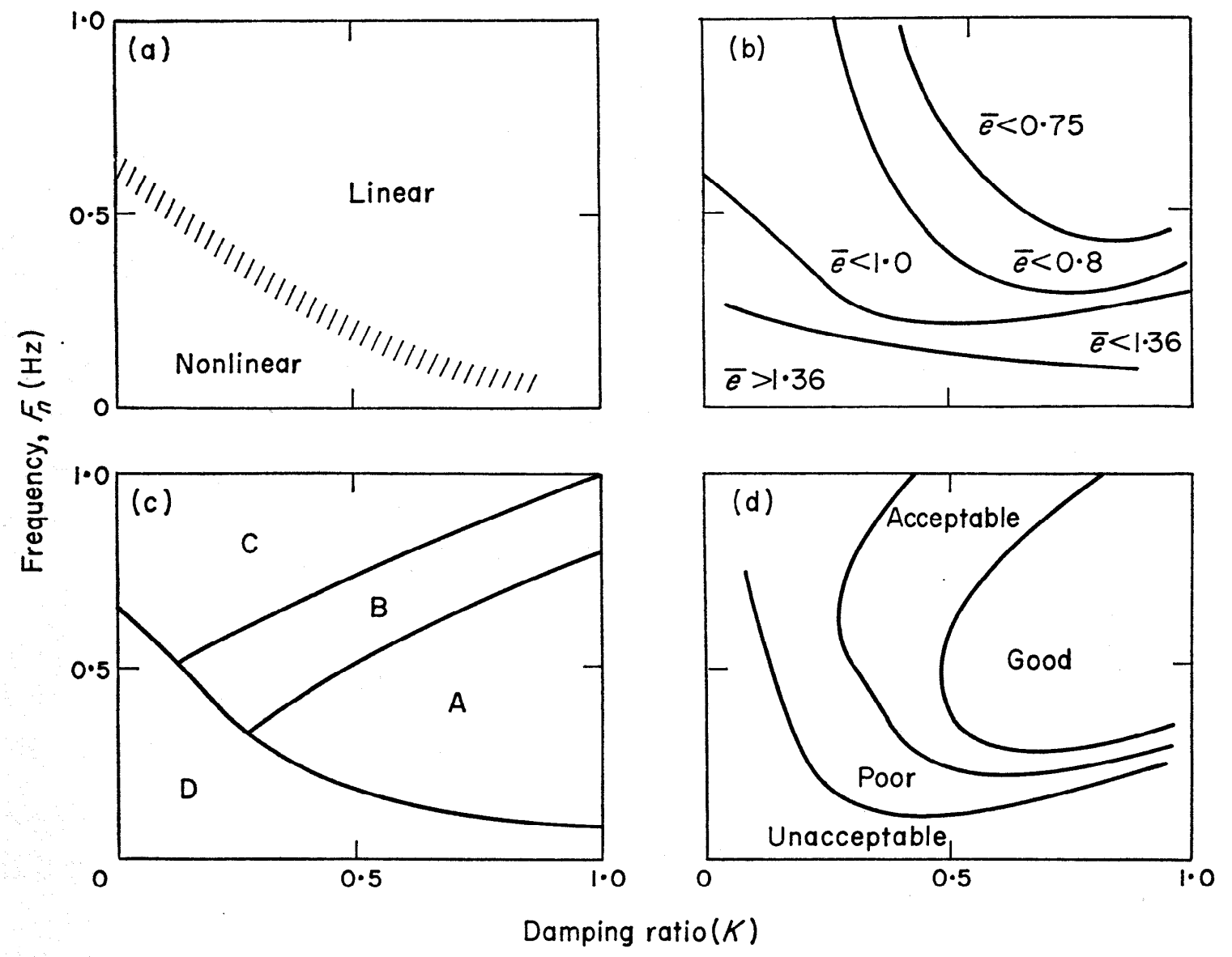

Figure 2. Results of experiments on linear modelling (Hall, 1963)

$$
\begin{array}{ll}
\text { Region } & \text { Form of model, K(s) (Hall, 1963) } \\
\text { A } & 0.16(1+0.67 \mathrm{~s})^{\mathrm{e}-0.2 \mathrm{~s}} /(1+0.2 \mathrm{~s}) \\
\text { B } & 0.25 \mathrm{~s}^{\mathrm{e}-0.2 \mathrm{~s}} \\
\text { C } & 0.5(1+0.77 \mathrm{~s})^{\mathrm{e}-0.2 \mathrm{~s}} /(1+2.5 \mathrm{~s}) \\
\text { D } & 0.0625(1+2 \mathrm{~s})^{\mathrm{e}-0.2 \mathrm{~s}} /(1+0.5 \mathrm{~s})^{2} \\
& \text { Where } \mathrm{K}(\mathrm{s})=(\text { stick output }(\text { in. })) /(\text { error signal }(\mathrm{deg} .))
\end{array}
$$

Table 1. Various forms of model for the human controller

Hall asked the experienced pilots who acted as experimental subjects to rate the controlled elements for its "handling qualities" as if it were an aircraft and the consensus of these ratings is shown in Figure 2(d). It is from comparison of plots (d) and (a) that the main justification for the utility of linear models of the human operator in the aircraft industry is derived-Hall states it thus: 
"If the pilot's opinion is 'good' the pilot is acting linearly . . . if a system is studied which has to be altered so that the pilot opinion will be high, the better the configuration becomes in terms of handling qualities the more accurate a linear analysis will be."

This is a very fair, and a virtually complete, assessment of the utility of linear models of the human controller, emphasizing the restricted, but useful, conditions under which they apply.

One final question which Hall considered was to suppose that the operator was acting linearly and determine the "source" of the remnant term, $n(t)$. Records of the pilot response, $m(t)$, for configurations in Region D of Figure 2(c) showed that superimposed on the output predicted by the linear model was a "rather frantic switching" mode, with the pilot alternating "somewhat arbitrarily between the two hard over stick" positions. Hall reports that the switching did not occur regularly and was not correlated with error zero crossings - he matches the remnant component in spectral density with a random telegraph waveform having a mean time between switching of 3-5 sec. Diamantides (1958) has reported a similar effect under the same conditions, and ascribes it to pilots attempting to obtain "informative feedback" about the controlled element dynamics by injecting a signal into the loop. He also reports that the injected signal is more apparent with less skilled pilots, and, in one operator at least, the signal disappeared with learning as an "exponential function of time."

\subsection{Utility of linear models in human operator studies}

Because of the strong theoretical constraints upon the circumstances under which the describing function is meaningful, several workers have studied the validity of the necessary assumptions in experiments with the human operator, and the extent to which overall behaviour, such as stability, may be predicted from the measured linear models. Elkind \& Darley (1963) measured the deviations from a Gaussian distribution of the operator's output, $m(t)$, the remnant, $n(t)$, and the error signal, $\mathrm{e}(\mathrm{t})$, for a controlled element, $\mathrm{G}$, which was a pure gain, with an input $\mathrm{r}(\mathrm{t})$ which was band-limited Gaussian noise. They report that the output "obtained with all inputs and the error and remnant signals obtained with medium bandwidth inputs appear to be approximately normally distributed." Hall (1957), in a similar experiment but with a controlled element of the form given in the equation for G(s) above, found that the amplitude distribution of the error signal was approximately normal, but the distribution of the operator's output appeared rectangular and even bimodal when the band-width and damping of the controlled element were low.

Jex, Cromwell \& Siskind (1960) and Smith (1963) have compared the stability boundary for the human operator, computed from describing function measurements, with the actual boundary found by experiment. They used the results obtained by Krendel \& McRuer (1960) to predict the stability of the controlled system for second-order unstable dynamics (negative damping ratio), and find it necessary to introduce an input-predictive mode of operation to account for the experimental results. Skolnick (1966) has used measured data on the human operator describing function to determine "capability bounds" on the human controller, and has proposed techniques

for optimizing the performance of a control system containing a human operator using these bounds.

Various workers have studied the effect of verbal instructions to an operator on the parameters of a corresponding linear model, and these effects are summarized by McRuer \& Krendel (1957) in what is still the most comprehensive and detailed discussion of linear modelling and its relevance 
to human operator studies. McRuer \& Krendel used two sets of instructions, one of which emphasized "speed" in reducing the effect of disturbance. For one operator they found no change in the measured describing function (d.c.) under the two conditions, whereas for another they found a distinct constant when the emphasis was on "speed"-lower d.c. gain and smoothing time-constant triple what it was for "speed" when the emphasis was on accuracy. Russell (1951) measured the change in linear models parameters after the operator had drunk a substantial quantity of alcohol, and reported lower d.c. gain and greatly reduced capability to introduce lead, that is, to estimate error-velocity.

Sheridan (1960) has used a technique for measuring the describing function on-line to follow changes in the model parameters when those of the controlled element undergo a step variation. He reports that, "the experienced operator adapts almost instantaneously if the parameters of controlled process or the type of display suddenly change." This is in accord with the more recent studies of Young, Green, Elland \& Kelly (1964) who investigated the time taken for the operator to adapt to changes in the gain of the controlled element, and sense of the error, in a simple compensatory tracking task. They report that, "adaptation generally occurs in 0.4 to 0.5 sec following a controlled element change, and the resulting error is usually reduced to its asymptotic level in 1 to $3 \mathrm{sec}$ following transition." Krendel \& McRuer (1960) have outlined a developmental approach to the learning of a tracking skill in terms of the parameters of the describing function at various stages. Fuchs (1962) has put forward a "progression-regression" hypothesis suggesting that in learning the parameters of the higher time derivatives of the error will be gradually given more weight, whilst under stress their relative weights will be reduced. DeLessio \& Palin (1961) put forward a program to identify the time-variation of the parameters of an operator's describing function, and hence form "an adaptive model for the human operator", but this program has not been carried out.

Briggs (1964) has defended the linear describing function as a powerful methodology for human operator studies, but claims that there has been too much effort expended on the development of the technique, and too little on "the descriptive quantification of behaviour and analytic tests of hypotheses about behaviour"; he fears that the same mistake will be made in future developments of nonlinear models. This is an important criticism and in the following section some details of the relationship between actual behaviour and its equivalent linear model are examined, both for their relevance to the utility of linear models and for their wider implications.

\subsection{Nature of the linear approximation and constraints upon it}

Controlled elements whose parameters vary progressively, but rapidly, over a range of values may be used to obtain records of the behaviour of the same operator under different conditions within a short span of time, and serve to illustrate some of the characteristics of the describing function discussed in Section 3.2. Figure 3 shows the input, $r(t)=\sin (\pi t / 5)$, a sine-wave of 10 seconds period, and the operator's output, for a tracking task with continuous manual input and continuous visual display of error, and controlled element dynamics of the form:

$$
\mathrm{G}(\mathrm{s})=\mathrm{L} / \mathrm{s}(\mathrm{s}+\mathrm{I} / \mathrm{T})^{2}
$$

that is a second-order lag of time-constant, $\mathrm{T}$, followed by a pure integration; this is similar to Hall's dynamics, with $\mathrm{k}=1$ and $\mathrm{w}_{\mathrm{n}}=1 / \mathrm{T}\left(\mathrm{F}_{\mathrm{n}}=1 / 2 \pi \mathrm{T}\right)$.

In the left part of the figure, which shows the response for a short time lag ( $\mathrm{T} \sim 0.25 \mathrm{sec}$ ), the operator's response has an overall shape which is similar to that of the sine-wave input but 
lagging it in phase. This response is clearly made up of a number of discrete movements, however, and is not the continuous sinusoid response which would be obtained from a linear servo in the same situation. If the Fourier transform of this response, however, it will clearly be found to have a very high percentage of its energy in the expected sinusoid, and very little in the "perturbation" due to the discrete movements. The converse is true of the response at high lags ( $\mathrm{T} \sim 0.7 \mathrm{sec}$ ), shown in the right part of Figure 3 . The operator is now responding so rapidly with such large amplitude movements that his output appears closer to a pulse-width modulated signal than a sinusoid. A Fourier transform would still show a phase-lagging signal at the input frequency, but this is now lower in amplitude and accounts for a minority of the energy in the response.
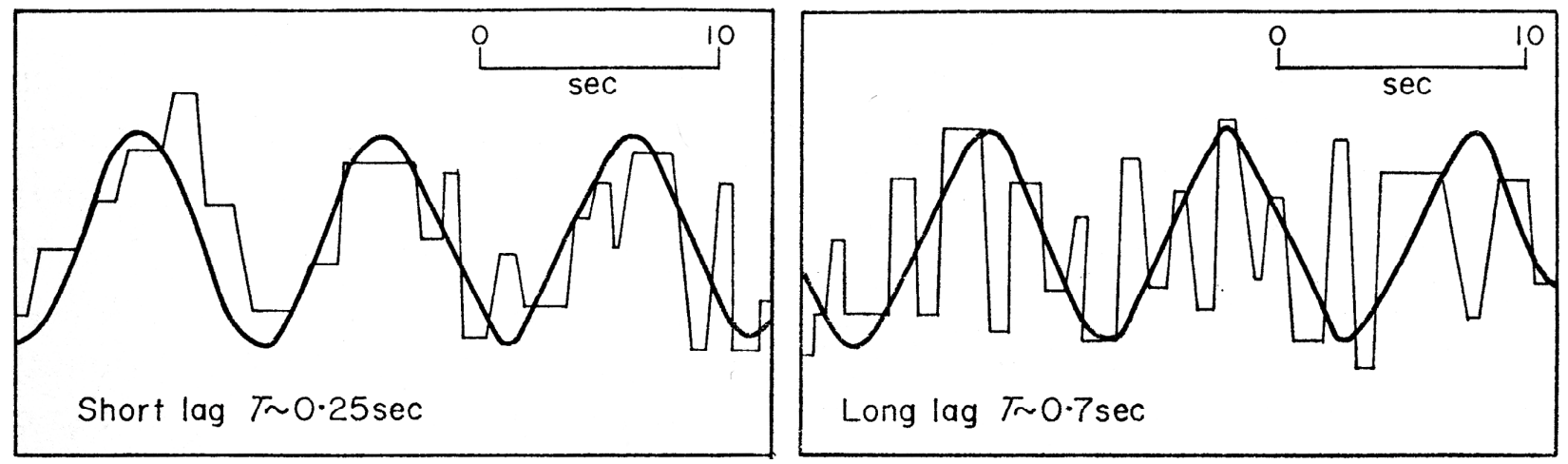

Figure 3. Sine wave tracking through cascaded lags

A correlational analysis of the control behaviour partially shown in Figure 3 would result in a good linear fit to the controller at short lags and a bad fit (high remnant) at long lags. More importantly, the model would differ greatly for the two situations, and yet it is plausible, both from an examination of the records and from the fact that they were taken within a few seconds of one another from the same operator, that the operator has not changed his control strategy in the least. This is the gravest defect of the describing function - that the linear model of an operator may vary widely as function of his environment without his control strategy changing at all.

The intermittency and discreteness of the human operator's response is not a newly discovered phenomenon-Telford (1931) reported a "refractory phase" in the motor responses to two stimuli presented within an interval of about $0.5 \mathrm{sec}$ of one another, and Craik (1947) described the type of response in a tracking task, shown in Figure 3, as "intermittent corrections" consisting of "ballistic movements." However, whilst many workers have followed up Telford's discoveries of a central refractory period in simple discrete stimulus/response situations, lack of development of both the theoretical and technological tools has made it impossible to go further with Craik's analysis until recently. Even now only a few steps forward have been taken, and no comprehensive and complete structure, equivalent to the describing function, is available for nonlinear studies of the human operator. In the following section, work on nonlinear models of the human operator is reviewed for its relevance to improved models of human adaptive behaviour. 


\section{Nonlinear Models of the Human Controller}

The evidence for a fundamental discrete-time, discrete-action basis for human perceptual-motor skilled behaviour has been presented in general reviews by Summers \& Ziedman (1964), Bekey (1965), Young \& Stark (1965) and Poulton (1966), and also in theses proposing sampled-data models of the human controller (Bekey, 1962; Lange, 1965). Definitive evidence has been gathered of discrete-action and discrete-time phenomena in peripheral behaviour such as hand and eye movements, and theoretical models of these have been explored in depth. Less firm evidence has been adduced for discrete-time phenomena in perception and decision-making, but no models have yet been proposed which can account for all the experimental data. In the following section work on discrete behaviour in substructures of the human controller is reviewed, whilst further sections outline sampled-data and "bang bang" models of overall tracking behaviour.

\subsection{Discrete phenomena in human peripheral dynamics}

In moving his hand from one position to another, or in rotating his eye from one fixation to another, the human operator has to vary the location of a mass using the force exerted by his muscles which is limited in its maximum value. Dynamically, the hand or eye is virtually a pure mass, with low dissipation of energy through friction, and low storage of potential energy through spring-like behaviour. A simple servomechanism, in controlling the location of an object, applies a force to it proportional to the deviation of the location from the desired one, in such a direction as to reduce the deviation. Bushaw (1953) showed that the control policy of the linear servomechanism was not time-optimal, in that it did not reduce the error in location to zero as rapidly as possible, and he showed that a "bang bang" controller, applying maximum available force in one direction for half the time and then applying it in the other, gave improved performance. From 1953 onwards, a number of workers proved, with increasing generality, that the minimum-time control of a linear system was achieved by a controller which applied either maximum or zero force (Fuller, 1960a). This result has been extended to the optimization of performance criteria other than settling time, such as error-functionals (Fuller, 1960b).

In 1962, Smith (1962) and Wilde \& Wescott (1962) published papers giving experimental evidence that the human operator used "bang bang" control in moving his hand and arm, and at the same time van der Gon, Thuring \& Strackee (1962) described a "handwriting simulator" which accurately reproduced the movements of the hand in writing using "bang bang" controllers in two dimensions. In a later paper, van der Gon \& Thuring (1965) reported that the controllers worked at a fixed force within a movement pattern, rather than at constant maximum force. They state that, "to write the same word involves the use of the same timing and that the instruction of change of size is interpreted as change of force"; since the size of the writing varies as the square of the force, small changes in force are adequate to produce large changes in size. Equally, the human operator does have an ultimate limit in the force applicable, and loading the hand or arm with more inertia reduces the speed of movement (Smith, 1962). The minimum time of application of the force for an unloaded limb was found by all workers to be about 90 msec, which tallies with the response-time of the muscle servomechanism (Hammond, Merton \& Sutton, 1956) and the rate at which nervous pulses are sent to the muscle (Lippold, Redfearn \& Vuco, 1957). A similar discrete action servomechanism has been discovered in the control of eye movements (Stark, Vossius \& Young, 1962; Young \& Stark, 1963), with independent control of 
positional saccades and velocity pursuit motion, again with forces applied for about $90 \mathrm{msec}$ in turn.

Apart from the clearly defined discrete phenomena in human limb and eye movements, there is considerable circumstantial evidence for discrete phenomena, "data-sampling" or a "psychological moment" in perception itself. Experiments on the "psychological refractory period" (Welford, 1952), on choice reaction times (Hick, 1952), on temporal numerosity (White, 1963), on periodicities in simple reaction times (Stroud, 1954; Augenstine, 1954; Venables, 1960), on backward masking of one stimulus by a succeeding one (Kolers, 1962), and on the reaction time to the cessation of a repetitive stimulus (Callaway \& Alexander, 1962), all suggest that visual perception is not a continuous process. Various authors have suggested, on the basis of such data, that the brain works in terms of a moment of time, in duration about $90 \mathrm{msec}$, within which events are confused in their temporal relationships. As Kolers (1968) and Allport (1968) have pointed out, however, no simple model of such discreteness in time can account for more than a minority of the known phenomena, although it is clear that some form of discontinuity is present. Some workers (Wiener, 1948; Lindsley, 1952; Surwillo, 1963) have attempted to link the hypothesized periodicity in perception of $90 \mathrm{msec}$ with the similar periodicity in the alpha rhythm of the brain, and indeed Surwillo has described definite experimental evidence of a strong correlation between alpha period and simple reaction time over a population. However, no incontrovertible evidence of such a link has been obtained.

\subsection{Sampled data models for human tracking behaviour}

The evidence for temporal discontinuities in human perception and movement, together with the observed nonlinearities in human tracking behaviour (Craik, 1947; Hick, 1948; Poulton, 1962) when perception and movement are coupled closely together, has lead to a number of proposals for data-sampling models of the human controller in which the display is sensed intermittently and a motor-pattern released according to what is observed. In control engineering (Kalman \& Bertram, 1959; Jury, 1958) such sampled-data control systems became of practical importance with the use of digital computers in control loops, and for the case where the sampling frequency is constant a theory of linear sampled data systems has been developed based on the z-transform, which is similar in power to the theory of continuous linear systems based on the Laplace transform. Because such a theory exists, it has been customary to base recent human operator models on sampled-data systems with constant sampling-frequency, although this does not fit the experimental data (Lange, 1965), and attempts have been made to develop techniques to deal with more complex sampling criteria (Bekey, 1962).

The earliest sampled-data model was that of North (1952) who took Tustin's model of the human operator and replaced the differential equations by difference equations. North matched the behaviour of his model against that of the human operator in terms of power spectra only, and the first study in which the behaviour was matched in the time domain was that of Ward (1958). More recently Bekey (1962), Lange (1965) and Kreifeldt (1965) have proposed sampled-data models for human tracking behaviour, and Bekey (1965) has reviewed some of this work. Lange's work was a continuation of the work of Wilde and Lemay (Wilde \& Wescott, 1962; Lemay \& Wescott, 1962), and has the most detailed experimental backing; the main points of his model and results are outlined in the following paragraph.

Lange considered compensatory tracking through a simple gain, with continuous manual control and visual display, of zero-mean Gaussian noise with a cut-off frequency of $3.8 \mathrm{rad} / \mathrm{sec}$, and used 
highly trained operators as subjects. In his model, the operator samples both position and velocity of error instantaneously, regularly at about $150 \mathrm{msec}$ intervals, and attempts to reduce both to zero by a bang bang output to actuate his hand. The qualitative nature of the output of the model is a far better match to the operator's output than that of a linear model. Quantitatively, correlations of between 0.8 and 0.9 were obtained between model errors and operator errors, corresponding to cross correlations between their outputs of between 0.98 and 0.99 . The match in the time-domain could have been improved by taking a varying sampling interval, and Lange suggests an extended model with random variation of the sampling frequency.

\section{3. "Bang bang” models of human controller for high-order systems}

Data-sampling models of the human control provide a good representation when the controlled element is a pure gain and the operator is effectively required to match a difficult waveform. In this situation, the movement of the hand to match the waveform, and the movement of the eye to track it, are clearly the main variables, and the tracking models are closely related to those of the hand and eye alone. The situation is also a very natural one, to which hand and eye co-ordination should have become well-suited during the course of evolution, and it is not surprising that the movement time of the eye, the reaction time delay between visual stimulus and motor response, and the movement time of the hand, are all similar in magnitude at about $180 \mathrm{msec}$ - it would be no advantage to the system to have one very much less than the others. Hence, a "sampling interval" of the same order is a reasonable approximation in these simple situations. When the lags in the controlled element become very much greater than those in the operator, however, the eye and hand in themselves become of less importance, and the problem-solving capability of the brain in between them comes to dominate the behaviour.

It was noted in Section 4.1 that "bang bang", or maximal force, control is the optimum strategy for the control of a pure second-order system which approximates to the dynamics of an eye or limb. This result has been extended to the time-optimal control of any linear system, and the maximum-effort controller is becoming as ubiquitous in the literature as the linear controller (Fuller, 1960a, 1962). Pew (1963) and Young \& Meiry (1965) presented experimental evidence that in the control of both stable and unstable second-order systems the human operator adopts a "bang bang" control strategy, and have shown that tracking improves if this strategy is forced upon the operator by giving him a two-position only control.

Because the two-level output of a "bang bang" controller is far simpler to monitor than the continuous output of a linear controller, and the control strategy can be represented by those points in the state-space of the controlled element at which the controller changes from one output value to the other (the "switching-line" in the position/velocity "phase-plane" for a second-order system), it is comparatively simple to measure the control policy of the human operator working in a "bang bang" mode. In particular, the adaption of the control policy during learning is readily followed, and since a plot of individual decision-points is obtained as a function of time it is possible to clarify the effects of indeterminacy in the policy (in the "search" phase), indeterminacy in the measurement of the policy (since only a limited number of data points are available), and time-variation of the policy with learning. In linear modelling by correlational techniques, the smoothing of data over time causes these factors to be inextricably mixed.

Li, Young \& Meiry (1965) have described qualitatively the variation of the human operator's switching line in learning to control an unstable second-order system. Weir \& Phatak (1967) 
have measured the time-variation of the switching line in response to step changes in the controlled element dynamics. However, as yet, there does not appear to have been published any detailed study of the learning of a high-order control skill, where a "bang bang" control policy is either forced by the nature of the controls, or expected to appear.

\subsection{Adaptive nonlinear models of the human controller}

Subject to the criteria of Section 2, any one model which gives a good match to the behaviour of the human operator is as legitimate as any other. Thus, the behaviour of many of the automatic adaptive controllers and learning systems described in the control and artificial intelligence literature (Tou \& Wilcox, 1964; Donaldson \& Kishi, 1967; Andreae, 1969) might be used to "model" that of the human operator. This would be done in a manner exactly analogous to that used with linear models: given a criterion for "goodness of fit", the modeller would vary the parameters of the model to obtain the value which best satisfied the criterion. Because linear models provide such a poor fit to the detailed output of the human operator, it is not surprising that their parameters have no natural interpretation in terms of learning variables and do not give rise to any useful model of human tracking behaviour which includes learning. Conversely, because of the strong quantitative and qualitative resemblances between the output of sampleddata and "bang bang" models and that of the human operator, these seem to offer a firmer foundation on which to build extensive models of human skilled behaviour.

The most advanced automatic learning systems have had a decision-making, rather than a continuous control, structure, and their outputs have been discrete "decisions" rather than analogue variables. This type of output is most similar to that of the human operator using a "bang bang" control mode, and it is reasonable to expect that useful models of human control behaviour might be obtained first, with the learning models available, when the controlled element is a high-order system with long lags, or the manual control is discrete in action.

Angel \& Bekey (1968) have described a simple finite-state machine for the control of a pure second-order system, based on experimental studies of discrete actuation in human limb movements (Section 4.1), which provides a qualitative match to many of the characteristics of human hand motion, and has self-adjusting properties giving it an adaptive capability; so far, they have not presented studies of the goodness of fit of the model to human tracking behaviour and its adaptation. Preyss \& Meiry (1968) have described a "stochastic model" of human learning behaviour in controlling a pure second-order system, in which the output is bang bang and its polarity is switched on the basis of probabilistic estimates of the efficacy of so doing. These estimates are themselves built up from prior experience using Bayes rule (Minsky \& Selfridge, 1961) to weight the evidence obtained from sensors giving quantized position and velocity information from the controlled element. This model learns to control the second-order system, and its behaviour both in tracking and in learning, is qualitatively similar to that of the human operator-again, no detailed analysis of goodness of fit is presented.

Gaines (1967) and Gaines \& Quarmby (1968) have presented comparative studies of human and machine learning behaviour, in which the learning model was an adaptive-threshold logic pattern-classifying adaptive controller, and report a close relationship between the effect of variation of the parameters of task difficulty on human and machine learning. Studies of learning system models of the human operator are currently limited only by the available suitable learning systems in a utilizable form. As more learning machines become generally available, preferably 
as computer programs for small, on-line process control machines, it will be possible to evaluate their utility as human operator models.

\subsection{Tracking with nonlinear controls}

Since the human operator of high-order systems adopts a strongly nonlinear control policy, it is of interest to consider whether his performance is enhanced through the use of a control which naturally induces this type of policy; for example, a two or three position joystick rather than a continuously variable control. Young \& Meiry (1965) have noted that, in high-order systems, the error is dependent on the integral of the control movement, and the operator must keep track of this quantity. With a continuous control this involves integration of a continuous function of time; with a two or three position controller, it involves summation of the time intervals when the output is positive, and subtraction of those for which it is negative; for a pulsing controller, which gives out fixed-duration, fixed height pulses, either positive or negative in sign, it involves only counting the excess of one type of pulse over the other. Thus these three types of control should be successively easier to use, provided the integrations in the system are adequate to filter out the quantization noise of the nonlinear controls.

Pew (1963) found in his studies that the performance of the human operator in controlling a pure second-order system was similar with a continuous joystick and a two-position switch. Kilpatrick (1964) found that when the controlled element dynamics were of the form, $1 / \mathrm{s}^{2}$ or $1 / \mathrm{s}^{2}(\mathrm{~s}+3)$, there was no significant difference between the two types of controller, whereas with a very difficult controlled element, requiring more lead, such as $1 / \mathrm{s}^{2}(\mathrm{~s}+1)$, the r.m.s. error for the continuous control was 50\% higher than for the 'bang bang' control. Young \& Stark (1965) note, in Kilpatrick's studies, that "even though the operator uses the continuous controller in a more or less "bang bang" fashion, he is able to use the "bang bang' controller in a pulse control fashion."

Gaines $(1966,1967)$ has reported that the use of pulsing controls not only improves the performance of the human operator in high-order systems, but is also less fatiguing. He was interested in obtaining a control for use in studies of training, which was itself difficult to use and involved interactions between the learning of the tracking task and learning to use the control. Building memory into the pulsing control system, such that the sign of a pulse obtained from one of two push-buttons depended upon that last pressed, gave a control with the required characteristics. The control consisted of a pair of push-buttons, one held in each hand, such that pressing one push-button would give out a positive impulse, whereas pushing the other would give out a negative impulse. The polarity of the two pushbuttons was not constant, however, and changed each time either was pressed. Hence, to obtain a stream of pulses of constant polarity, it is necessary to alternate between the two push-buttons.

Initially this control feels most awkward and unnatural to use, but eventually, after 10 to 30 minutes of use under reasonable conditions, it becomes as simple and natural to use as the nonreversing push-buttons. The problems in using this control may be appreciated by considering the situation in which the operator has pushed a button and the error has increased-his natural tendency is to push the other button, but the correct response is to push the same button again. Gaines (1966) describes the various stages of learning to use the push-button controls, from an almost entirely verbal strategy, through the build-up of response structures, to a highly-skilled, non-verbalizable control strategy. 


\subsection{Control strategies in multi-variable situations}

Whilst much research effort has been devoted to the study of the dynamics of the peripheral mechanisms of the human controller, and to the linking of these by control strategies for compensatory tracking, and much progress has been made in the understanding and modelling of the human operator in simple situations, there is no comparable understanding of the control strategies adopted in more realistic situations, where the operator has multiple, diverse and interacting tasks to be performed either simultaneously, or sequentially. Hence models of the human controller in the simple tracking situations used in laboratory experiments may be very different from those of behaviour encountered in more realistic situations.

At the level of overall performance, there have been a number of studies of two-dimensional tracking tasks, with the error on one axis presented as the horizontal axis on an oscilloscope, and the error on the other presented as the vertical axis - the control being a two-dimensional, continuous-output joystick (Chernikoff, Duey \& Taylor, 1959; Duey \& Chernikoff, 1959; Chernikoff \& LeMay, 1963). The main result of these was that tracking in both axes deteriorated as the task dynamics in the two become more different, and that a two-dimensional task with the same dynamics in both axes was similar in difficulty to the equivalent one-dimensional task. At a similar conceptual level, Dander (1963) has investigated the possibility of predicting pilot ratings of multi-axis control tasks from single-axis data. The interference between widely differing tasks, which do not in themselves interact, has been extensively investigated in studies to improve the sensitivity of performance measures through the use of secondary tasks (Knowles, 1963). The factors which make "secondary loading" techniques useful operate to make the modelling and prediction of interference difficult, since there is generally a level below which a secondary task shows no detectable effect - it is using up the operator's "spare capacity."

At a detailed level, the main problem in modelling human control strategies in multi-variable situations is that of measuring and simulating "attention switching" as the operator multiplexes his control capabilities to various parts of the total system. One of the more accessible and important indications of attention is the instrument at which the operator is looking, and much effort has been devoted to measuring and modelling the human controller's visual behaviour in a many-instrument, multi-dimensional tracking task (Senders, 1964; Carbonell, 1966; Senders, Ward \& Carbonell, 1967). Senders originally proposed, and tested experimentally, a model in which the frequency of sampling an instrument was proportional to the potential information flow-rate through that instrument regarded as a communication channel. The later studies extend this to models which take account of the "queuing" of instruments for attention, and the risk taken in not reading a particular instrument.

Even pursuit tracking, where the operator is shown not only the error but also the input, or disturbing, signal, is itself a multi-variable tracking situation, and poses far greater difficulties in the analysis of the operator's behaviour than does compensatory tracking. Poulton (1952a, b, $1957 \mathrm{a}, \mathrm{b}$ ) has studied the differences between behaviour in compensatory and pursuit tracking in great detail, and suggests that the advantages of the pursuit situation stem from its enabling a complete separation to be made between the demanded input to the system, the "track", and the operator's own input through the system. This separation aids both the prediction of future system behaviour, and the modelling by the operator, as part of his learning process, of the demand signal and system dynamics. 
The pursuit tracking situation becomes even more complex when the operator can see not only the immediate value of the demand signal, but also some segment of its future values, for example, in a car-driving situation. Classical control theory gives no indication of how advantage may be taken of such a preview, and hence it has been impossible, until recently, to approach the modelling of human control behaviour in the vehicle-steering situation from a control-theoretical point of view. Sheridan \& Roland (1966) have now used the modern control technique of "Dynamic Programming" (Bellman \& Dreyfus, 1962) to obtain a normative model of the optimum control strategy in this situation for comparison with that of the human operator. This interesting and powerful approach has also been expounded in some detail by Thomas (1962), and offers the opportunity for a substantial advance in the understanding of human control behaviour. Dynamic programming is essentially a computational technique which enables very general control problems to be solved, given a criterion of optimality, by numerical algorithms. Its main disadvantage is the amount of computation and data storage required, but this is far less than that for a complete search of all possible control policies.

\section{Conclusions}

The present state of knowledge about the human controller is conveyed best in the previous subsection on multi-variable situations. Even the pursuit-tracking situation has not yet been adequately analysed or described, and this is still a "laboratory situation" far removed from the multi-variable realities of everyday life. The single-input, single-output, compensatory tracking situation is the only one which shows signs of yielding soon to the application of modern control theory and modern experimental technology. It is well to remember in any study of human behaviour that our imperfect knowledge itself only pretends to capture a small fraction of that behaviour.

However, in the context of previous knowledge of the human controller, there have been notable advances in recent years, and the non-linear models described in Section 4 have a greater credibility than that of past linear models. The detailed structure of the behaviour of these models can be seen (by the complex pattern-recognition process of the human eye) to be similar to that of human behaviour in similar circumstances, and this, at the present state of art, is as important as mere numerical "goodness of fit."

Psychology is full of examples of the danger of premature mathematization and ill-considered quantification of human behaviour. The mathematics and physics of each day and age have been applied to the contemporary behavioural sciences, only to be discarded as new branches of mathematics and physics have been opened up. It is not that behaviour cannot be quantified, but rather that it is too rich for full description and we always describe those, often minor, aspects for which we happen to have terms available.

It is safe to predict that the algebraic theory of semigroups and automata (Clifford \& Preston, 1961, 1967; Liapin, 1963; Booth, 1967; Wymore, 1967; Arbib, 1968) will come to play an increasingly important role in the behavioural sciences during the next decade. It is almost as safe to predict the decline and fall of many of their applications, as even more powerful and appropriate mathematical tools become-available; the first book on algebraic semigroups is only of 1960 vintage.

However, the ability of the digital computer to acquire and analyse vast quantities of data means that our knowledge of human behaviour, particularly in the simple situation of a compensatory 
tracking skill, is now growing very rapidly. It may also be expected that our knowledge of how to deal with such vast quantities of data will also increase, and that the models of human skilled behaviour, outlined in this paper, will become increasingly refined and more widely applicable. In particular, their domain will be extended from highly skilled military personnel to both the learning behaviour of unskilled individuals and to the pathological behaviour of, for example, brain-damaged individuals.

\section{References}

ALLPORT, D. A. (1968). Phenomenal simultaneity and the perceptual moment hypothesis. $\mathrm{Br}$. J. Psychol. 59, 395.

ANDREAE, J. H. (1969). Learning machines. In Encyclopaedia of Information, Linguistics and Control. London: Pergamon.

ANGEL, E. S. \& BEKEY, G. A. (1968). Adaptive finite-state models of manual control systems. IEEE Trans. MMS, 9, 15.

ARBIB, M. A. (Ed.) (1968). Algebraic Theory of Machines, Languages and Semigroups. New York: Academic Press.

AUGENSTINE, L. G. (1954). Evidences of periodicities in human task performance. In Information Theory in Psychology. Glencoe, III.: Free Press.

BARTLETT, F. C. (1958). Thinking. London: Allen and Unwin.

BEKEY, G. A. (1962). An investigation of sampled data models of the human operator in control systems. Ph.D. Thesis, University of California, and ASD-TDR-62-36.

BEKEY, G. A. (1965). Description of the human operator in a control system. In Modern Control Systems Theory. New York: McGraw Hill.

BELlMAN, R. E. \&DREYFUS, S. E. (1962). Applied Dynamic Programming. Princeton, N.J.: Princeton University Press.

BIRKHOFF, G. D. (1927). Dynamical Systems, Vol. IX. Providence, R. I.: Am. Math. Soc. Coll.

BOOTH, T. L. (1967). Sequential Machines and Automata Theory. London: Wiley.

BOOTON, R. C. (1953). The analysis of nonlinear control systems with random inputs. In Nonlinear Circuit Analysis II. Brooklyn, N.Y.: Polytechnic Inst. Brooklyn Press.

BRIGGS, G. E. (1964). The generality of research on transfer functions. In Categories of Human Learning. New York: Academic Press.

BUSHAW, D. W. (1953). Differential equations with discontinuous forcing terms. Stevens Tech. Inst., N.J., Rep. 469.

CALLAWAY, E. \& ALEXANDER, J. D. (1962). The temporal coding of sensory data-an investigation of two theories. J. Gen. Psychol. 62, 293.

CARBONELL, J. R. (1966). A queuing model of many-instrument visual sampling. IEEE Trans. HFE, 7, 157.

CHERNIKOFF, R., DUEY, J. W. \& TAYLOR, F. V. (1959). Two-dimensional tracking with identical and different control dynamics in each coordinate. USNRL Rep. 5424. 
CHERNIKOFF, R. \& LEMAY, M. (1963). Effect of various display-control configurations on tracking with identical and different co-ordinate dynamics. J. exp. Psychol. 66, 95.

ClifFORD, A. H. \& PRESTON, G. B. (1961). The Algebraic Theory of Semigroups I. Providence, R.I.: Am. Math. Soc.

ClifforD, A. H. \& PRESTON, G. B. (1967). The Algebraic Theory of Semigroups 11. Providence, R.I.: Am. Math. Soc.

CRAIK, K. J. W. (1947). Theory of the human operator in control systems. Br. J. Psychol. 38, 56.

DANDER, V. A. (1963). Predicting pilot ratings of multi-axis control tasks from single-axis data. IEEE Trans. HFE, 4, 15.

DELESSIO, N. X. \& PALIN, J. (1961). An adaptive model for the human operator. Polytechnic Inst., Brooklyn, PIBMRI-909-61.

DIAMANTIDES, N. D. (1958). A pilot analog for airplane pitch control. J. Aeronaut. Sci. 361.

DONALSON, D. D. \& KISHI, F. H. (1967). Review of adaptive control system theories and techniques. In Modern Control Theory. New York: McGraw Hill.

DUEY, J. W. \& CHERNIKOFF, R. (1959). The use of quickening in one coordinate of a twodimensional tracking system. USNRL Rep. 5428.

ELKIND, J. I. (1956). Characteristics of simple manual control systems. MIT Tech. Rep. 111.

ELKIND, J. I. \& DARLEY, D. L. (1963). The normality of signals and describing function measurements of simple manual control systems. IEEE Trans. HFE, 7, 115.

FUCHS, A. H. (1962). The progression-regression hypotheses in perceptual-motor skill learning. J. exp. Psychol. 63, 177.

FULLER, A. T. (1960a). Phase space in the theory of optimum control. J. Electron. Control, 8, 341.

FULLER, A. T. (1960b). Relay control systems optimized for various performance criteria: Proc. 1st. Int. Congr. IFAC, Moscow.

FULLER, A. T. (1962). Bibliography of optimum nonlinear control of determinate and stochastic-difinite systems. J. Electron. 13, 590.

GAINES, B. R. (1966). Teaching machines for perceptual-motor skills. Proc. Programmed Learning Conf. Loughborough. In Aspects of Educational Technology. London: Methuen (1967).

GAINES, B. R. (1967). Automated feedback trainers for perceptual-motor skills. Final Report M.o.D. Contract.

GAINES, B. R. \& QUARMBY, D. J. (1968). The convergence of adaptive pattern classifiers in control systems. Dept. Electrical Engineering Science, University of Essex, RM 68/2.

GIBSON, J. E. (1963). Nonlinear Automatic Control. New York: McGraw Hill.

VAN DER GON, J. J. D. \& THURING, J. Ph. (1965). The guidance of human writing movements. Kybernetik, 2, 145. 
VAN DER GON, J. J. D., THURING, J. PH. \& STRACKEE, J. (1962). A handwriting simulator. Physics Med. Biol. 6, 407.

HALL, I. A. M. (1957). Effects of controlled element on the human pilot. Aero. Eng. Lab., Princeton University, Rep. No. 389.

HALL, I. A. M. (1963). Study of the human pilot as a servo element. J. R. Aeronaut. Soc. 67, 351.

HAMMOND, P. H., MERTON, P. A. \& SUTTON, G. G. (1956). Nervous gradation of muscular contraction. Br. Med. Bull. 12, 214.

HICK. W. E. (1948). The discontinuous functioning of the human operator in pursuit tasks. Q. $J$. Exp. Psycho, 1, 36.

HICK, W. E. (1952). On the rate of gain of information. Q. J. Exp. Psychol. 4, 11

JEX, H. R., CROMWELL, C. H. \& SISKIND, R. K. (1960). Correlation of experimental and theoretical limits for pilot control of unstable second order systems, Syst. Tech. Inc., Calif., U.S.A.

JURY, E. I. (1958). Sampled-data Control Systems. New York: Wiley.

KALMAN, R. E. \& BERTRAM, J. E. (1959). A unified approach to the theory of sampling systems, J. Franklin Inst. 267, 405.

KILPATRICK, P. S. (1964). Comparison of relay and manual controllers for systems with high order dynamics, B.S. Thesis, MIT.

KNOWLES, W. B. (1963). Operator loading tasks. Hum. Factors, 6, 155.

KOLERS, P. A. (1962). Intensity and contour effects in visual masking. Vision Res. 2, 227.

KOLERS, P. A. (1968). Some psychological aspects of pattern recognition. In Recognizing Patterns. Cambridge, Mass.: MIT Press.

KREIFELDT, J. G. (1965). A sampled-data pursuit tracking model. IEEE Trans. HFE, 6, 65.

KRENDEL, E. S. (1951). A preliminary study of the power spectrum approach to the analysis of perceptual-motor performance. Wright Patterson Air Force Base, Ohio, Tech. Rep. 6723.

KRENDEL, E. S. (1952). The spectral density study of tracking performance. Wright Patterson Air Force Base, Ohio, TR-52-11.

KRENDEL, E. S. \& McRuER, D. T. (1960). A servo-mechanisms approach to skill development. J. Franklin Inst. 269, 24.

LANGE, G. W. (1965). Syntheses of a model of the human operator engaged in a tracking task. Ph.D. Thesis, University of London.

LEMAY, L. P. \& WESCOTT, J. H. (1962). The simulation of human operator tracking using an intermittent model. IRE Int. Congr. Human Factors in Electronics.

Ll, Y. T., YOUNG, L. R. \& MEIRY, J. L. (1965). Adaptive functions of man in vehicle control systems. Proc. IFAC Symp., Teddington, U.K.

LIAPIN, E. S. (1963). Semigroups. Providence, R.I.: Am. Math. Soc. 
LICKLIDER, J. C. R. (1960). Quasi-linear models in the study of manual tracking. In Developments in Mathematical Psychology. Glencoe, III.: Free Press.

LINDSLEY, D. B. (1952). Psychological phenomena and the electro-encephalogram. EEG Clin. Neurophysiol. 4, 443.

LIPPOLD, O. C. J., REDFEARN, J. W. T. \& Vuco, J. (1957). The rhythmical activity of groups of motor units in the voluntary contraction of muscle. J. Physiol. Lond. 137, 473.

LURIA, A. (1961). The Role of Speech in the Regulations of Normal and Abnormal Behaviour. Oxford: Pergamon Press.

MCRUER, D. T., GRAHAM, D., KRENDEL, E. S. \& REISENER, W. (1965). Human pilot dynamics in compensatory systems. AFFDL-TR-65-15.

MCRUER, D. T. \& KRENDEL, E. S. (1957). Dynamic response of human operators. WADCTR-56-523.

MCRUER, D. T. \& KRENDEL, E. S. (1959). The human operator as a servo system element. J. Franklin Inst. 267, 381-403, 511-536.

MELTON, A. W. (Ed.) (1964a). Categories of Human Learning. New York: Academic Press.

MELTON, A. W. (1964b). The taxonomy of human learning-an over-view. In Categories of Human Learning. New York: Academic Press.

MILLER, G. A., BREGMAN, A. S. \& NORMAN, D. A. (1965). The computer as a general purpose device for the control of psychological experiments. In Computers in Biomedical Research, Eds. R. W. Stacy and B. Waxman. New York: Academic Press.

MINSKY, M. \& SELPRIDGE, O. G. (1961). Learning in random nets. In Information Theory. London: Butterworths.

MIRSKY, L. (1955). Linear Algebra. Oxford: Clarendon Press.

NORTH, J. D. (1952). The human transfer function in servo systems. In Automatic and Manual Control. London: Butterworths.

PEW, R. W. (1963). Temporal organisation in skilled performance. Ph.D. Thesis, University of Michigan.

POULTON, E. C. (1952a). Perceptual anticipation in tracking with two-pointer and one-pointer displays. Br. J. Psychol. 43, 222.

POULTON, E. C. (1952b). The basis of perceptual anticipation in tracking. Br. J. Psychol. 43, 295.

POULTON, E. C. (1957a). On the stimulus and response in pursuit tracking. J. exp. Psychol. 53, 189.

POULTON, E. C. (1957b). Learning the statistical properties of the input in pursuit tracking. J. Exp. Psychol. 54, 28.

POULTON, E. C. (1962). A source of nonlinearity in pursuit tracking. In Complex Vehicular Controls, a Symposium at Inst. Aviation Medicine, Farnborough, U.K. 
POULTON, E. C. (1966). Tracking behaviour. In The Acquisition of Skill, Ed. E. A. Bilodeau. New York: Academic Press.

PREYSS, A. E. \& MEIRY, J. L. (1968). Stochastic modelling of human learning Behaviour. IEEE Trans. MMS, 9, 36.

QUARMBY, D. J. (1968). The influence of the environment on the convergence of a learning machine. Electronics Letters.

RIESZ, F. \& Sz. NAGY, B. (1955). Functional Analysis. Frederick Ungar.

RUSSELL, L. (1951). Characteristics of the human as a linear servo element. M.S. Thesis, MIT.

SENDERS, J. W. (1964). The human operator as a monitor and controller of multi degree of freedom systems. IEEE Trans. HFE, 5, 2.

SENDERS, J. W., WARD, J. L. \& CARBONELL, J. R. (1967). Human visual sampling processes - a simulation validation study. Final Rep. Contract NAX-1-5059, Bolt Beranek and Newman.

SHERIDAN, T. B. (1960). Experimental analysis of time variation of the human operator's transfer function. Proc. 1st Int. Congr. IFAC, Moscow.

SHERIDAN, T. B. \& ROLAND, R. D. (1966). A normative model for control of vehicle trajectory in an emergency maneuver. Ann. Meeting Highway Research Board, Washington, DC.

SKOLNICK, A. (1966). Stability and performance of manned control systems. IEEE Trans. HFE, 7, 115.

SMITH, O. J. M. (1962). Nonlinear computations in the human controller. IRE Trans. BME, 9, 125.

SMITII, R. H. (1963). On the limits of manual control. IEEE Trans. HFE, 4, 56.

STARK, L., Vossius, G. \& YOUNG, L. R. (1962). Predictive control of eye tracking movements. IRE Trans. HFE, 3, 52.

STROUD, J. M. (1954). The fine structure of psychological time. In Information Theory in Psychology. Glencoe, III.: Free Press.

SUMMERS, L. G. \& ZIEDMAN, K. (1964). A study of manual control methodology with annotated bibliography. NASA-CR 125.

SURWILLO, W. W. (1963). The relation of simple response time to brain-wave frequency and the effects of age. EEG Clin. Neurophysiol. 15, 105.

TELFORD, C. W. (1931). The refractory phase of voluntary and associated responses. J. Exp. Psychol. 14

THOMAS, R. E. (1962) Development of new techniques for analysis of human controller dynamics. MRL-TDR 52-65.

TOU, J. T. \& WILCOX, R. H. (1964). Computer and Information Sciences. New York: Spartan Books. 
TUSTIN, A. (1947). The nature of the human operators response in manual control and its implication for controller design. J. Instn. Elect. Engrs, 94, 190.

VENABLES, P. H. (1960). Periodicity in reaction time. Br. J. Psychol. 51, 37.

WARD, J. R. (1958). The dynamics of a human operator in a control system. Ph.D. Thesis, University of Sydney, Australia.

WARD, J. L. \& SENDERS, J. W. (1966). Methodological studies of tracking behaviour-the effect of various supplemental information feedbacks, Final Rep. Nonr 4605(00), Bolt Beranek and Newman.

WEIR, D. H. \& PHATAK, A. V. (1967). Model of human operator response to step transitions in controlled element dynamics. NASA CR-671.

WELFORD, A. T. (1952). The 'psychological refractory period' and the timing of high-speed performance; a review and theory. Br. J. Psychol. 43, 2.

WHITE, C. T. (1963). Temporal numerosity and the psychological unit of duration. Psychol. Monogr. 77(12).

WIENER, N. (1948). Cybernetics. New York: Wiley.

WILDE, R. W. \& WESCOTT, J. H. (1962). The characteristics of the human operator engaged in a tracking task. Automatica, 1, 5 .

WYMORE, A. W. (1967). A Mathematical Theory of Systems Engineering. New York: Wiley.

YOUNG, L. R. \& STARK, L. (1963). Variable feedback experiments testing a sampled data model for eye tracking movements. IEEE Trans. HFE, 38, 51.

YOUNG, L. R., GREEN, D. M., ELKIND, J. I. \& KELLY, J. A. (1964). Adaptive dynamic response characteristics of the human operator in simple manual control. IEEE Trans. HFE, 5,6 .

YOUNG, L. R. \& STARK, L. (1965). Biological control systems-A critical review and evaluation. NASA CR-190.

YOUNG, L. R. \& MEIRY, J. L. (1965). Bang bang aspects of manual control in high-order systems. IEEE Trans. AC, 10, 336. 